Research Paper

\title{
Role of glutathione biosynthesis in endothelial dysfunction and fibrosis
}

\author{
Cristina Espinosa-Díez ${ }^{\mathrm{a}, 1}$, Verónica Miguel ${ }^{\mathrm{a}, 1}$, Susana Vallejo ${ }^{\mathrm{b}}$, Francisco J. Sánchez ${ }^{\mathrm{a}}$, \\ Elena Sandoval $^{\mathrm{a}, 2}$, Eva Blanco ${ }^{\mathrm{a}}$, Pablo Cannata ${ }^{\mathrm{c}}$, Concepción Peirób ${ }^{\mathrm{b}}$, Carlos F. Sánchez-Ferrer ${ }^{\mathrm{b}}$, \\ Santiago Lamas ${ }^{\mathrm{a}, *}$ \\ a Department of Cell Biology and Immunology, Centro de Biología Molecular "Severo Ochoa", (CSIC-UAM), Madrid, Spain \\ ${ }^{\mathrm{b}}$ Department of Pharmacology, Faculty of Medicine, Universidad Autónoma de Madrid and Instituto de Investigación Sanitaria Hospital Universitario La Paz (IdiPAZ), \\ Spain \\ c Department of Pathology, Instituto de Investigaciones Sanitarias-Fundación Jiménez Díaz, Universidad Autónoma de Madrid, Spain
}

\section{A R T I C L E I N F O}

\section{Keywords:}

Glutamate-cysteine ligase

ROS

Glutathione

Endothelial dysfunction

Kidney Fibrosis

\begin{abstract}
A B S T R A C T
Glutathione (GSH) biosynthesis is essential for cellular redox homeostasis and antioxidant defense. The ratelimiting step requires glutamate-cysteine ligase (GCL), which is composed of the catalytic (GCLc) and the modulatory (GCLm) subunits. To evaluate the contribution of GCLc to endothelial function we generated an endothelial-specific Gclc haplo-insufficient mouse model (Gclc e/+ mice). In murine lung endothelial cells (MLEC) derived from these mice we observed a 50\% reduction in GCLc levels compared to lung fibroblasts from the same mice. MLEC obtained from haplo-insufficient mice showed significant reduction in GSH levels as well as increased basal and stimulated ROS levels, reduced phosphorylation of eNOS (Ser 1177) and increased eNOS S-glutathionylation, compared to MLEC from wild type (WT) mice. Studies in mesenteric arteries demonstrated impaired endothelium-dependent vasodilation in $\operatorname{Gclc}(e /+)$ male mice, which was corrected by pre-incubation with GSH-ethyl-ester and $\mathrm{BH}_{4}$. To study the contribution of endothelial GSH synthesis to renal fibrosis we employed the unilateral ureteral obstruction model in WT and $\operatorname{Gclc}(e /+)$ mice. We observed that obstructed kidneys from $\operatorname{Gclc}(e /+)$ mice exhibited increased deposition of fibrotic markers and reduced Nrf2 levels. We conclude that the preservation of endothelial GSH biosynthesis is not only critical for endothelial function but also in anti-fibrotic responses.
\end{abstract}

\section{Introduction}

Endothelial dysfunction constitutes a major challenge from both the biological and clinical standpoints as it is fundamentally associated to the vascular damage present in major pathological entities such as hypertension, diabetes, atherosclerosis, and aging [1]. An important body of literature supports a causative link between oxidative distress and impaired vascular function [2,3]. Since the discovery of endothelium-derived relaxing factor (EDRF), later identified as nitric oxide (NO), overwhelming evidence has been mounted regarding the disturbed redox state of endothelial cells and therefore their inability to maintain adequate vasodilation. At the cellular level, antioxidant responses to injury are elicited through multiple pathways. Among these pathways, the endogenous nucleophile glutathione (GSH) is of paramount importance for redox homeostasis, due to its high intracellular concentrations $(1-10 \mathrm{mM})$, its capacity to interact with peroxidases and its potential to act as an electron donor for free radicals [4-6]. GSH synthesis proceeds through two major steps regulated by the enzymes glutamate cysteine ligase (GCL) and glutathione synthetase (GS). GCL is the rate-limiting enzyme in the biosynthesis of GSH. GCL has been the object of intense study regarding the regulation of its expression and activity [7]. GCL is a heterodimeric holoenzyme that includes two regulatory domains, the catalytic (GCLc, $73 \mathrm{kDa}$ ) and the modifier (GCLm, $33 \mathrm{kDa}$ ) subunits; each of them is the object of distinct regulation $[8,9]$. The interaction between the two subunits and that of both of them together with their common substrates, cysteine and glutamate, dictates the final efficiency of GSH synthesis [10].

Dysregulation of GSH synthesis has been described in several

\footnotetext{
Abbreviations: Ach, acetylcholine; ARE, antioxidant response element; $\mathrm{BH}_{4}$, Tetrahydrobiopterin; DMNQ, 2,3-Dimethoxy-1,4-naphthoquinone; EDRF, Endothelium-derived relaxing

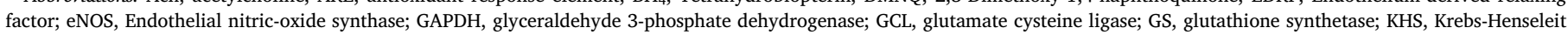

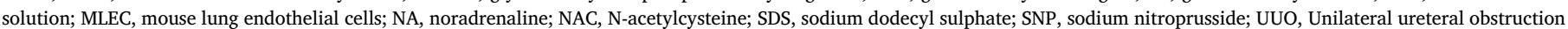

* Correspondence to: Centro de Biología Molecular "Severo Ochoa" (CSIC-UAM), Nicolás Cabrera 1, 28049 Madrid, Spain.

E-mail address: slamas@cbm.csic.es (S. Lamas).

${ }^{1}$ Contributed equally.

${ }^{2}$ Permanent address: Departamento de Biología Celular y Molecular, Centro Universitario de Ciencias Biológicas y Agropecuarias, Universidad de Guadalajara, Mexico.
} 
pathological conditions including liver injury [11], diabetes [12], neurological disorders [13], organ fibrosis [14] and cardiovascular disease [15]. Human polymorphisms of GCLc and/or GCLm may also result in decreased GSH synthesis and severe pathological phenotypes $[16,17]$. Genetic ablation of $\mathrm{Gclm}$ in mice is associated with impaired vascular reactivity [18], ovarian failure [19], lung inflammation [20] and mitochondrial dysfunction [21]. Of interest, the relationship between alterations in the redox status and fibrogenesis is intimately related to GSH homeostasis [14]. In this regard, it has been demonstrated that TGF- $\beta$ decreases the expression of GCL [22] and our group reported that a specific miRNA, miR-433 was in part responsible of this repression mechanism $[14,23,24]$. However, due to the lethal embrionary phenotype of Gclc null mice, the specific role of the vascular suppression of GCLc has been difficult to address in vivo. In this work, we present data regarding the generation and characterization of conditionally endothelial-deficient Gclc mice. We found that endothelial haplo-insufficiency is sufficient to promote endothelial dysfunction in mesenteric beds, leading to eNOS inactivation in endothelial cells of heterozygous mice. In addition, these mice express reduced levels of the master regulator of antioxidant defense, Nrf2 and are prone to renal fibrosis.

\section{Material and methods}

\subsection{Generation of endothelial-specific Gclc $(e /+)$ Knockout Mice}

Tie2-Cre transgenic mice were obtained from The Jackson Laboratory (Bar Harbor, ME). We generated endothelial-specific Gclc knockout $[\operatorname{Gclc}(e /+)]$ mice by intercrossing $G c l c(f / f)$ mice with Tie2Cre transgenic mice. The floxed Gclc mouse line, described in [25] was provided under agreement by Professors Ying Chen and Vasilis Vasiliou, from the University of Colorado. We conducted all studies on littermates. Animals were handled in agreement with the Guide for the Care and Use of Laboratory Animals contained in Directive 2010/63/EU of the European Parliament.

\subsection{PCR analysis}

For differentiation of the $G c l c(f)$ versus $G c l c(+)$ allele, genomic DNA isolated from tails was analyzed and genotyping was confirmed by PCR analysis using specific primers for DNA amplification (Sigma, St. Louis, MO) as described in [25]. The Gclc(f) allele was detected using primers A (GGGTGTTGG GTCGTTTGT) within the NEO gene, and B (CTATAATGTCCTGCACTGGG) within intron 3. The $\operatorname{Gclc}(e)$ allele was detected using primers C (TAGTGAACGGTGTTAAAGG) within intron 3, and D (TCACTGGATTCT CTCACC) within intron 6 . The $G c l c(w t)$ allele was detected using primers B and C. Primers (GCGGTCTGGCAGTAAAAACTATC, forward) and (GTGAAACAGC ATTGCTGTCACTT, reverse) were used to detect the Cre transgene.

\subsection{Isolation of mouse lung endothelial cells (MLEC) and primary lung fibroblasts}

Mice were sacrificed by cervical dislocation and lungs were excised and digested with collagenase from Clostridium histolyticum (Sigma, St. Louis, MO). The mixed population obtained was subjected to positive selection with anti-ICAM-2 (BD Biosciences, San Jose, CA, USA) and anti-IgG-coated magnetic beads (Invitrogen, Carlsbad, CA, USA); the leftover mixed cell population (fibroblast and macrophages) was subjected to negative selection with anti-FCsRII/III (BD Biosciences, San Jose, CA, USA). MLEC were cultured in EGM2 media and grown on a mixture of fibronectin (Sigma, St. Louis, MO), type I collagen (Thermo Scientific, Rockford, IL, USA), and $0.1 \%$ gelatin (Sigma, St. Louis, MO)coated plates. Primary lung fibroblasts were cultured in Dulbecco's Modified Eagle's medium (DMEM) with 10\% Fetal bovine serum supplementation.

\subsection{Western blot and densitometry analysis}

After treatment, cells were washed with phosphate-buffered saline (PBS) and lysed in $150 \mu \mathrm{l}$ RIPA lysis buffer containing $150 \mathrm{mM} \mathrm{NaCl}$, $0.1 \%$ sodium dodecyl sulphate (SDS), $1 \%$ sodium deoxycholate, $1 \%$ NP40 and $25 \mathrm{mM}$ Tris- $\mathrm{HCl} \mathrm{pH} \mathrm{7.6,} \mathrm{in} \mathrm{the} \mathrm{presence} \mathrm{of} \mathrm{protease} \mathrm{(Complete,}$ Roche Diagnostics, Mannheim, Germany) and phosphatase inhibitors (Sigma-Aldrich, St. Louis, MO). Cells were harvested by scraping, samples were clarified by centrifugation at $13,000 \mathrm{rpm}$ for $15 \mathrm{~min}$ at $4{ }^{\circ} \mathrm{C}$ and protein concentration was measured with the BCA assay (Thermo Scientific, Rockford, IL, USA). Equal amounts of protein (40-80 $\mu \mathrm{g})$ from the total extract were separated by electrophoresis using an acrylamide/bisacrylamide (10\%) gel and transferred to a nitrocellulose membrane (GE Healthcare, Germany) at $12 \mathrm{~V}$ for $20 \mathrm{~min}$ in a semi-dry Trans-Blot Turbo system (Bio-Rad, Hercules, CA). After blocking the membranes in 5\% skimmed milk in TBS-T $(10 \mathrm{mM})$, they were incubated with the appropriate primary antibodies, anti-GCLc (generous gift of Dr. Kavanagh's laboratory), anti-GCLm (1:1000; sc32251, Santa Cruz Biotechnology, CA, USA), anti- $\alpha$ SMA (1:1000; sc32251, Santa Cruz Biotechnology, CA, USA), anti- $\beta$-actin antibody (1:15000; A1978, Sigma, St. Louis, MO), anti-tubulin (1:15000; T9026, Sigma, St. Louis, MO), anti-eNOS (1:1000; 610297, BD Biosciences, San Jose, CA, USA) and anti-p-eNOS (Ser1177) (1:1000; 9571, Cell Signaling Technology, Boston, USA). Secondary antibodies against rabbit and mouse were from LI-COR Biosciences (Lincoln, NE). Densitometry of at least 3 Western blots for each experiment was done using $\beta$-actin or glyceraldehyde 3-phosphate dehydrogenase (GAPDH) (1:15000; Sigma, St. Louis, MO) as housekeeping controls for the total levels of protein loaded. Phospho eNOS signals were corrected by the levels of total eNOS, after normalization with $\beta$-actin. These analyses were represented in bar graphs showing the mean \pm SEM of protein respective to control conditions.

\subsection{Real-time PCR}

Total RNA was isolated with miRNeasy Mini Kit (Qiagen, California, USA). Reverse transcription was performed with $1 \mu \mathrm{g} / \mu \mathrm{l}$ mRNA sample using iScriptTM cDNA Synthesis Kit (Bio-Rad, Hercules, CA). qRT-PCR was carried out with the $\mathrm{iQ}^{\mathrm{TM}} \mathrm{SYBR}$ Green Supermix (Bio-Rad, Hercules, CA), using specific primers for mRNA amplification (Sigma, St. Louis, MO). The relative quantification of gene expression was determined using the $2^{-\Delta \Delta \mathrm{Ct}}$ method [26]. Using this method, we obtained the fold changes in gene expression normalized to an internal control gene, GAPDH. The sequences of the primers were: mouse GAPDH, forward: $5^{\prime}-$ GAGTCAACGGATTTGGTCGT-3', reverse: 5'-TTGATTTTGGAGG GATCTC G-3'; mouse FN, forward: 5'-AGAGGCTGTGTTGCTGTGAA-3', reverse: 5'-AA ATCCATCGGGTATCTGGA-3'; mouse Coll $\alpha 1$, forward: 5'TGG AGAGGAAAGTGGCGGGGAG-3', reverse: 5'-GCCTCACGGAACCACGAACG-3'; mouse GCLm, forward: 5'-CGGACGACCTGGTGAGAGA-3', reverse: 5'-CATTGTGTCCCCTAATGCCTT-3'; mouse NRF2, forward: 5'GTGTTGGG AATGGTCGTGGGGAATG-3', reverse: 5'-CCAATGCCACGGCCATAGCAGTA GC-3'; mouse $\alpha$-SMA, forward: $5^{\prime}$-CTGACAGAGGCACCACTGAA-3', reverse: 5'-CATCTCCA GAGTCCAGCACA-3'.

\subsection{GSH assay}

Reduced and oxidized forms of GSH were measured in MLEC utilizing the luminescence GSH-Glo and 2GSH/GSSG assays, based on the conversion of a luciferin derivative into luciferin in the presence of GSH (Promega, Wisconsin, USA) according to the manufacturer's instructions. The cells were analyzed on a luminescence plate reader (Glomax, Promega, Wisconsin, USA).

\subsection{ROS production}

MLEC were plated in a 96-well plate to reach a confluence of $70 \%$. 
The cells were treated with or without $10 \mu \mathrm{M}$ of Cyclosporine A (Sigma, St. Louis, MO) for $2 \mathrm{~h}, 25 \mu \mathrm{M}$ 2,3-Dimethoxy-1,4-naphthoquinone (DMNQ) (Sigma, St. Louis, MO) for $1 \mathrm{~h}$ or $200 \mu \mathrm{M} \mathrm{H}_{2} \mathrm{O}_{2}$ (Sigma, St. Louis, MO) for $10 \mathrm{~min}$ at $37^{\circ} \mathrm{C}$. The cells were then stained with $5 \mu \mathrm{M}$ of CellROX $^{\circledR}$ Deep Red Reagent (ThermoFisher Scientific, Waltham, MA) by adding the probe to the complete medium and incubating the cells at $37^{\circ} \mathrm{C}$ for $30 \mathrm{~min}$. The cells were then washed with PBS and analyzed on a fluorescence plate reader using the following wavelengths: emission 665 nM, excitation 644 nM (Glomax, Promega, Wisconsin, USA).

\subsection{Immunoprecipitation assay}

MLEC from $\operatorname{Gclc}(\mathrm{e} /+)$ and $\operatorname{Gclc}(\mathrm{f} / \mathrm{f})$ were seeded into $100 \mathrm{~mm}$ culture dishes to reach a confluence of 70\% and deprived of FBS for $16 \mathrm{~h}$ before treatments. They were treated with or without GSNO $50 \mu \mathrm{M}$ for $1 \mathrm{~h}$. Cells were lysed in RIPA lysis buffer, harvested by scraping and samples were cleared by centrifugation at $13,000 \mathrm{rpm}$ for $15 \mathrm{~min}$ at $4{ }^{\circ} \mathrm{C}$. Protein A/G-coupled agarose beads (Thermo Scientific, Rockford, IL, USA) were pre-incubated with anti-eNOS antibody (1:10; 610297, BD Biosciences, San Jose, CA, USA) for $1 \mathrm{~h}$ at $4{ }^{\circ} \mathrm{C}$ and then incubated for $2 \mathrm{~h}$ with cell lysate at $4{ }^{\circ} \mathrm{C}$. IgG1 $\mathrm{\kappa}$ antibody (1:10, 401401, Biolegend, San Diego, CA, USA) was used in the negative control condition. After incubation, eNOS immunoprecipitation product was washed three times with cold PBS buffer. $1 \times$ SDS loading buffer was used to elute eNOS for immunoblotting analysis of eNOS S-glutathionylation, using primary antibodies against eNOS (1:1000; 610297, BD Biosciences, San Jose, CA, USA) and GSH (1:1000; 101-A, Virogen, Watertown, NY, USA).

\subsection{Vascular reactivity}

The animals were briefly exposed to a chamber filled with carbon dioxide until they fell unconscious and then immediately sacrificed by cervical dislocation. The mesentery was removed, and placed in a Petri dish containing Krebs-Henseleit solution (KHS) (KHS; composition: $\mathrm{NaCl} 115, \mathrm{CaCl}_{2} 25, \mathrm{KCl} 4.6, \mathrm{KH}_{2} \mathrm{PO}_{4} 1.2, \mathrm{MgSO}_{4} .7 \mathrm{H} 2 \mathrm{O} 1.2, \mathrm{NaHCO}_{3}$ 25, glucose 11.1 and $\mathrm{Na}_{2}$ EDTA 0.03 .) at $4{ }^{\circ} \mathrm{C}$. The third branch mesenteric arteries were dissected from $G C L c(f / f)$ and $G C L c(e /+)$ mice (mean internal diameter ranged between 200 and $400 \mu \mathrm{m}$ ). The arteries were cleaned free of fat and connective tissue under a light microscope and mounted as ring preparations on a small vessel myograph [27] to measure isometric tension. Arteries were bathed in KHS at $37{ }^{\circ} \mathrm{C}$ continuously bubbled with a $95 \% \mathrm{O}_{2}-5 \% \mathrm{CO}_{2}$ mixture, which yields a $\mathrm{pH}$ of 7.4 and their passive tension and internal circumference were determined. The arteries were subjected to optimal tension ( $90 \%$ of the tension equivalent to an intramural pressure of $100 \mathrm{~mm} \mathrm{Hg}$. After $30 \mathrm{~min}$ of stabilization, the vessels were exposed to $125 \mathrm{mmol} / \mathrm{L} \mathrm{KCL}$ for $2 \mathrm{~min}$ in order to check their functional integrity. Segments failing to achieve a maximum active tension equivalent to a pressure of $100 \mathrm{mmHg}$ were rejected for experimental purposes.

The arteries were contracted with the concentration of $1 \mu \mathrm{mol} / \mathrm{L}$ noradrenaline (NA), required to produce approximately $80 \%$ of the maximum response. Contractility to NA was not statistically different among groups. Relaxation response to acetylcholine (ACh) was subsequently assessed by adding cumulative concentrations of the vasodilator at $2 \mathrm{~min}$ intervals (final bath concentrations $10 \mathrm{nmol} / \mathrm{L}$ to $10 \mu \mathrm{mol} / \mathrm{L}$ for ACh). Some microvessels from male mice were pre-incubated with increasing concentrations of tetrahydrobiopterin $\left(\mathrm{BH}_{4}\right)$ $(10 \mu \mathrm{M}) 120 \mathrm{~min}$ before and during the administration of NA and ACh. Other vascular segments were treated with $10 \mu \mathrm{M}$ N-acetylcysteine (NAC) and $10 \mu \mathrm{M}$ GSH-ethyl-ester for $30 \mathrm{~min}$ in advance and during the administration of NA, and ACh, respectively. Finally, in selected experiments, instead of $\mathrm{ACh}$, concentration-dependent curves to the endothelium-independent vasodilator sodium nitroprusside (SNP; $1 \mathrm{nmol} / \mathrm{L}$ to $10 \mu \mathrm{mol} / \mathrm{L}$ ) were performed.

\subsection{Unilateral ureteral obstruction (UUO)}

Studies were conducted according to the NIH Guide for the Care and Use of Laboratory Animals. Male $G c l c(f / f)$ and $\operatorname{Gclc}(e /+)$ mice (12-14week-old) were anesthetized, the left ureter was ligated, and the kidney was obstructed ( $n=6$ per group), using the contralateral kidney as control (non-obstructed), as previously described [28]. Animals were sacrificed at 10 days after surgery. Kidneys were perfused in situ with cold saline before removal. Half a kidney was snap frozen in liquid nitrogen for RNA and protein studies.

\subsection{Renal histology}

At the end of the UUO procedure, both kidneys were perfused with PBS and fixed in $4 \%$ neutral buffered formalin for $24 \mathrm{~h}$. Sections of 3$\mu \mathrm{m}$ thickness were stained with Sirius red as described previously [29]. Fibrosis analysis was defined as the percentage of Sirius Red area in the entire kidney section excluding perivascular staining and the evaluation was performed by a renal pathologist in a blind-folded fashion. Microphotographs were obtained using a digital camera (Nikon D3) connected to a Nikon's Eclipse TE2000-U light microscope (Nikon Instruments Europe B.V., Badhoevedorp, The Netherlands).

\subsection{Statistical analysis}

Statistical analysis was performed with GraphPadPrism (GraphPad Software). Data are expressed as means \pm SEM. Differences among groups with one experimental condition were assessed with Kruskal-Wallis test with Dunn's correction. Two-way ANOVA with Bonferroni correction was used to analyze differences among groups exposed to more than one condition. Differences between only two groups were analyzed with Mann-Whitney two-tailed test.

\section{Results}

\subsection{Generation of endothelial-specific Gclc $(e /+)$ knock-out mice and genotypic characterization}

To evaluate the contribution of endothelial GSH to vascular function we intercrossed Tie2-Cre $(+/+)$ mice, that express Cre recombinase specifically in the endothelium under the control of the Tie 2 promoter [30], with $G c l c(f / f)$ mice. In the latter, exons 4 and 6 of the Gclc allele are flanked by LoxP sites [25]. The possible genotypes to be obtained are depicted in Fig. 1. After several rounds of crossing we were able to generate endothelial haplo-insufficient Gclc mice, here termed Gclc(e/

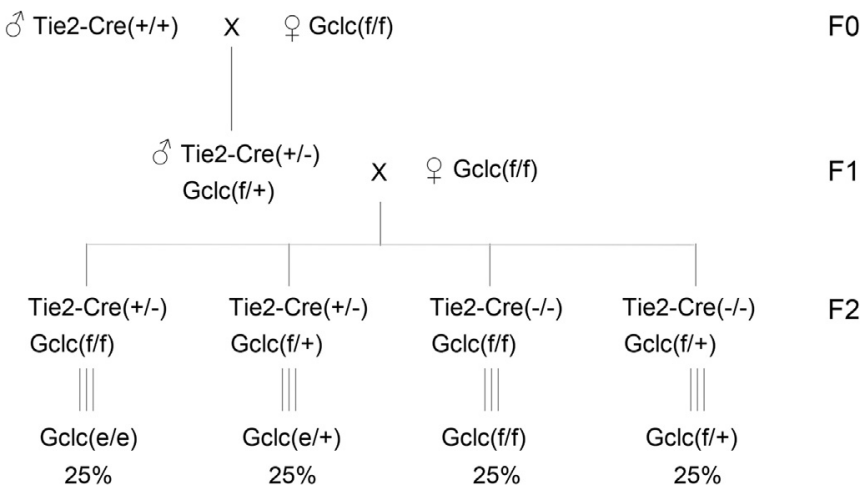

Fig. 1. Breeding scheme for generating specific Gclc endothelial null mice. Tie2-Cre $(+/+)$ male mice were crossed with female Gclc floxed $[G c l c(f / f)]$ mice to generate F1 generation. Male mice from F1 generation were crossed with female $G c l c(f / f)$ with the aim to obtain homozygous mice with endothelial-specific deletion of Gclc [Gclc (e/e)]. The expected Mendelian frequency of all 4 genotypes is indicated, though $G c l c(e / e)$ mice were not obtained. $\operatorname{Gclc}(e /+)$ endothelial haplo-insufficient mice were obtained and utilized in this study and $\operatorname{Gclc}(f / f)$ were used as controls. 
A

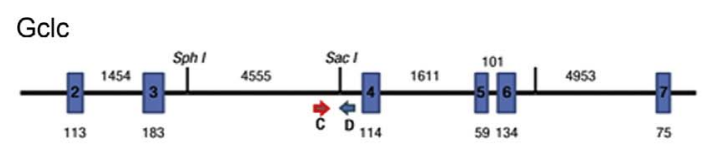

Allele

Targeting construct

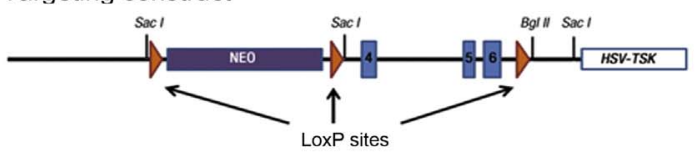

Targeted allele

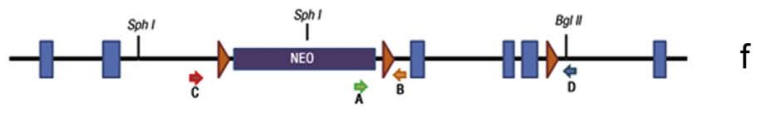

Targeted allele after recombination

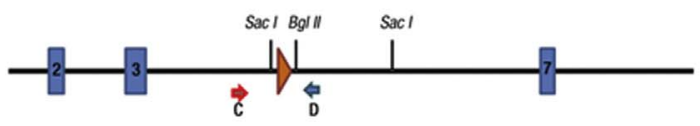

e

B

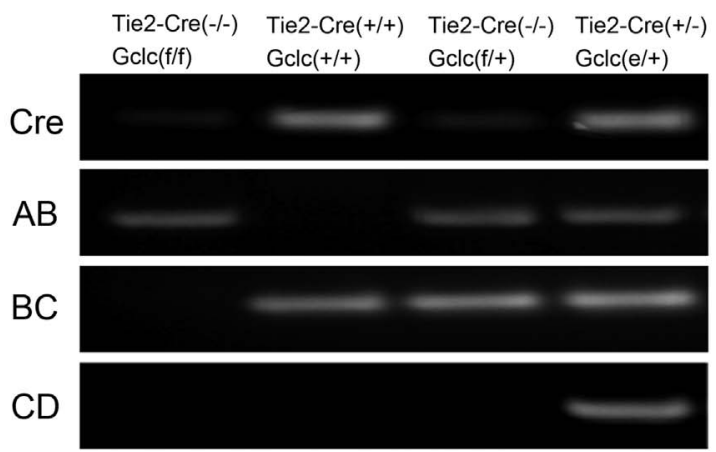

Fig. 2. Genetic validation of $G C L c(e /+)$ endothelial haplo-insufficiency. A) Schematic of the wild-type Gclc allele ( + ), targeting-construct targeted allele (f), and the deleted allele (e), following Cre-mediated recombination. NEO, neomycin-resistance mini-cassette, and HSV-TK, herpex simplex virus thymidine kinase mini-cassette, represent genes used as selectable markers. The positions of primers for PCR analysis are shown as arrows. B) PCR analysis of genomic DNA for the Tie2-Cre and Gclc alleles. The 203-pb BC, 200-pb AB and 180-pb CD bands represent the Gclc (+), Gclc (f) and Gclc (e) alleles, respectively.

+). In Tie2-Cre-positive Gclc(f/f) mice, we detected endothelial-specific recombination of the $\operatorname{Gclc(f)}$ allele, Gclc(e) allele by PCR using the primers indicated in Fig. 2A and described in the methods section. As expected, we did not detect the Gclc(e) allele in DNA isolated from tails of Gclc(f/f) mice lacking Tie2-Cre (Fig. 2B, lane 1). However, we detected $G c l c(w t)$ allele in both, positive and negative Tie2-Cre mice (Fig. 2B, lanes 2 and 3). As shown in Fig. 2B, lane 4 we detected the presence of Gclc (wt) as well as Gclc (e/+), supporting that all Tie2-Cre positive mice are heterozygous. In spite of further attempts to generate homozygous mice lacking endothelial GCLc we were unable to breed these, due perhaps to embryonic or perinatal lethality associated to this phenotype [31].

\subsection{Effects of endothelial GCLc decrease on the redox state}

In order to characterize GCLc expression in endothelial cells, we isolated mouse lung endothelial cells and fibroblasts from $G c l c(f / f)$ mice and $\operatorname{Gclc}(\mathrm{e} /+)$ as indicated in Methods. GCLc protein expression was significantly downregulated in the endothelium from haplo-insufficient mice respect to the control mice. However, we did not observe significant changes in the modifier subunit GCLm (Fig. 3A), suggesting that there are no evident compensatory responses to the deficit of the catalytic subunit. The protein levels of GCLc and GCLm in lung fibroblasts were similar in the control and the haplo-insufficient mice, thus attesting to the specificity of the endothelial deletion (Fig. 3B).

To evaluate the functional relevance of GCLc reduction, we measured GSH levels in these cells. Gclc haplo-insufficiency was associated with a decrease in GSH levels by $80 \%$ respect to control mice (Fig. $4 \mathrm{~A}$ ). We did not detect significant changes in the abundance of the oxidized form of GSH, GSSG (data not shown). Exposure of MLEC from Gclc(e/ + ) to previously described electrophiles [32] as well as to $\mathrm{H}_{2} \mathrm{O}_{2}$, showed enhanced ROS generation compared to identically treated control MLEC (Fig. 4B). GSH depletion and increased oxidative stress trigger endothelial dysfunction and eNOS uncoupling [33]. The analysis of eNOS activation is a good indicator of vascular function; therefore we studied redox-dependent phosphorylation of Ser 1177 [34]. Whereas eNOS phosphorylation levels increased along time of exposure to $\mathrm{H}_{2} \mathrm{O}_{2}$ in $G c l c(f / f)$ endothelial cells, MLEC from haplo-insufficient mice did not show any significant time-dependent increase in eNOS phosphorylation (Fig. 4C). Of importance, MLEC from Gclc $(\mathrm{e} /+)$ exhibited enhanced eNOS S-glutathionylation in basal conditions (Fig. 4D), thus providing a biochemical basis for eNOS uncoupling [35]. Overall, these data support that endothelial depletion of GCLc levels results in significant perturbation of redox homeostasis, manifested in a reduced capacity to handle electrophilic stress and eNOS dysfunction.

\subsection{Haplo-insufficiency of the Gclc gene is associated with endothelial dysfunction in male mice}

To evaluate the consequences of Gclc haploinsufficiency on endothelial function we isolated mesenteric arteries from $\operatorname{Gclc}(\mathrm{f} / \mathrm{f})$ and $\operatorname{Gclc}(\mathrm{e} /+)$ mice and studied their reactivity as indicated in Methods. We first studied endothelial-dependent relaxation in vessels from Gclc (f/f) and $\operatorname{Gclc}(\mathrm{e} /+)$ male and female mice. $\operatorname{Gclc}(\mathrm{e} /+)$ male mice exposed to concentration-dependent relaxations evoked by acetylcholine (ACh) showed an impairment in the contractile response compared to control mice, whereas in female mice no significant differences were detected (Fig. 5A). No differences among groups in vascular relaxation elicited by sodium nitroprusside (SNP) were observed (Fig. 5B). Due to the obvious gender differences in vascular relaxation only male mice were chosen to perform the rest of the vascular reactivity studies.

\subsection{Endothelial dysfunction related to Gclc haplo-insufficiency is attenuated by tetrahydrobiopterin $\left(\mathrm{BH}_{4}\right)$ and $\mathrm{GSH}$ ethyl-ester}

To understand the mechanisms involved in endothelial dysfunction in $\operatorname{Gclc}(\mathrm{e} /+)$ mice, we pre-incubated mesenteric vessels from $G c l c(\mathrm{f} / \mathrm{f})$ and $\operatorname{Gclc}(\mathrm{e} /+)$ mice with agents potentially modulating GSH levels or eNOS function, namely NAC, the permeable analog of GSH, GSH ethylester (GSH-EE) and the eNOS cofactor tetrahydrobiopterin $\left(\mathrm{BH}_{4}\right)$. Pretreatment with $\mathrm{BH}_{4}$ also resulted in increased amelioration of the vascular dysfunction associated to Gclc haplo-insufficiency (Fig. 6A). Pretreatment of vessels with NAC was not accompanied by a significant improvement in vascular reactivity in Gclc (e/+) mice (Fig. 6B), whereas pre-treatment with GSH-EE was associated with increased relaxation in response to the higher concentrations of ACh (Fig. 6C). Taking together these results indicate that endothelial dysfunction of $\operatorname{Gclc}(\mathrm{e} /+)$ mice is associated with reduced GSH bioavailability that can be compensated by improving eNOS function through the addition of critical cofactors that limit or revert its uncoupled state.

\subsection{Haplo-insufficiency of endothelial Gclc results in a moderate renal fibrotic phenotype}

As described in previously published work GCLc and GCLm are significantly downregulated after induction of renal fibrosis using the unilateral ureteral obstruction (UUO) model [23]. To evaluate if the reduction of GCLc basal levels present in the Gclc(e/+) mice could have an effect in renal fibrogenesis, we performed the UUO procedure in 
A

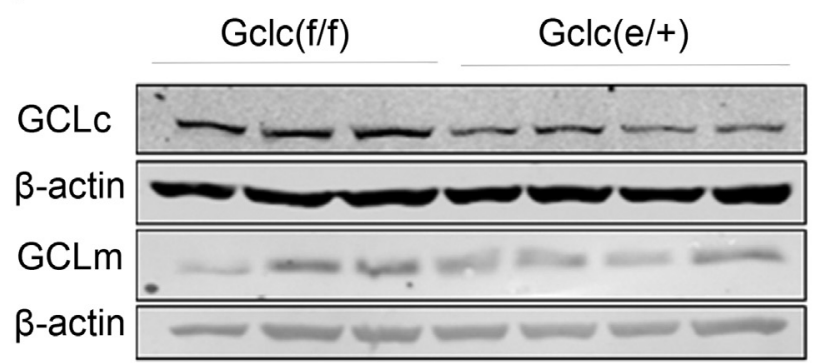

MLEC

B

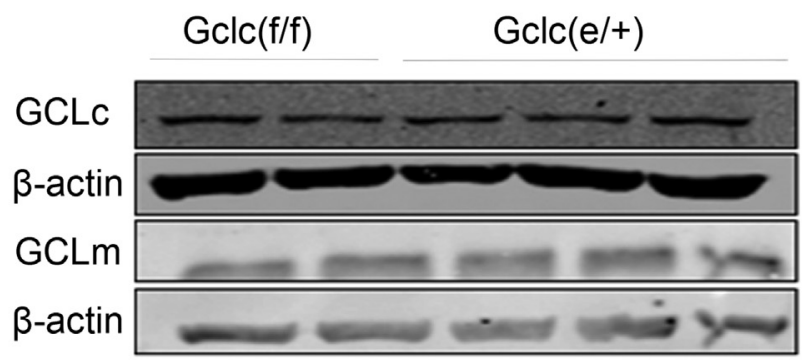

FIBROBLASTS

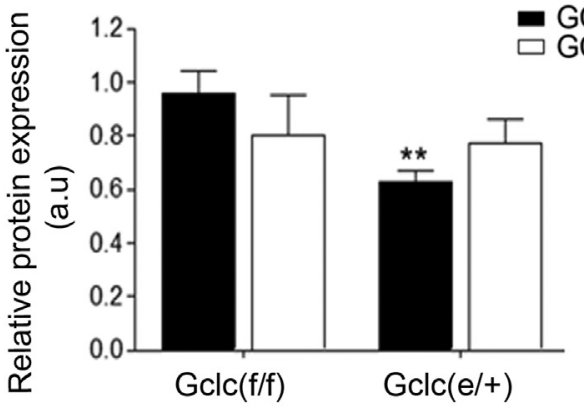

GCLC

GCLm

$\operatorname{Gclc}(f / f) \quad \operatorname{Gclc}(e /+)$

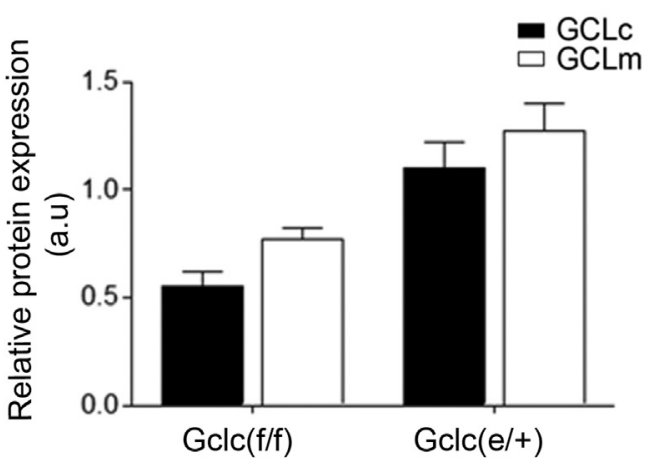

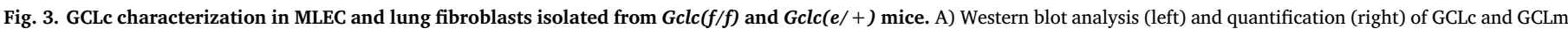

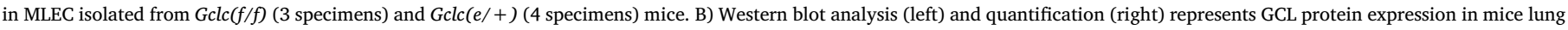

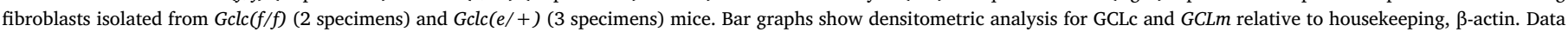
represent mean $\pm \mathrm{SEM},{ }^{* *} \mathrm{p}<0.01$ compared to GCLc from $G c l c(f / f)$ mice MLEC.

these mice and determined the abundance of several fibrosis markers. Even though the expression of GCLc was evaluated in whole kidney tissue and not exclusively in the vascular compartment, the levels of GCLc were reduced after the UUO procedure and this reduction was enhanced in $\operatorname{Gclc}(\mathrm{e} /+$ ) (Fig. 7A). The abundance of canonical fibrotic markers such as Col1a1 and fibronectin were also increased in Gclc haplo-insufficient kidneys (Fig. 7B-C). In contrast, although UUO promoted a dramatic increase in the mRNA or protein levels of $\alpha$-SMA, no significant differences between control and haplo-insufficient mice were detected (Fig. 7D-E).

We checked for the presence of a homeostatic redox response after the challenge of UUO. Of interest, there was no differential compensation of GCLm levels in Gclc(e/+) mice but in contrast, the UUO-dependent increase in the levels of Nrf2, the master regulator of antioxidant responses, was completely absent in the Gclc haplo-insufficient mice (Fig. 7F-G).

To evaluate the presence of kidney fibrosis, histological analysis with Sirius Red was performed in renal tissue from $3 \mathrm{WT}$ and $3 \mathrm{Gclc}(\mathrm{e} /$ + ) mice subjected to the UUO procedure. As shown in Fig. $8 \mathbf{A}$ the foldchange in the intensity of the Sirius Red stained areas was generally higher in the obstructed kidneys from the $\operatorname{Gclc}(e /+)$ animals. A representative micrograph of Sirius Red-stained renal tissue from one WT and one $\operatorname{Gclc}(e /+)$ is shown in Fig. 8B.

\section{Discussion}

In the present study we have studied the specific contribution of endothelial GSH to endothelial function. Using murine lung endothelial cells (MLEC) from endothelial-specific Gclc haplo-insufficient Gclc(e) + ) mice we have shown a reduction in GSH levels as well as increased
ROS levels, reduced phosphorylation of eNOS (Ser 1177) and increased eNOS S-glutathionylation. Further, we demonstrated that GSH deficiency impairs endothelium-dependent vasodilation in $\operatorname{Gclc}(e /+)$ male mice, which was corrected by pre-incubation with ethyl-ester GSH and $\mathrm{BH}_{4}$. In addition, we have also studied the contribution of endothelial GSH synthesis to renal fibrosis. Using the UUO model in WT and Gclc(e/ + ) mice we have shown that obstructed kidneys from Gclce/+) mice exhibited increased deposition of fibrotic markers and reduced Nrf2 levels. This report demonstrates that endothelial GSH constitutes a major protective mechanism in endothelial dysfunction and UUO-induced renal fibrosis.

GCLc is the rate-limiting enzyme for GSH biosynthesis. To evaluate the contribution of GCLc to endothelial function we generated endothelial-specific haplo-insufficient $\operatorname{Gclc}(e /+)$ mice. We also attempted to generate homozygous mice lacking endothelial Gclc but we were unable to breed them. It is conceivable that the complete genetic deficiency of Gclc leads to a lethal embryonic or perinatal phenotype as it has been previously described that complete deficient homozygosity of Gclc results in death before gestational day 13, whereas the $G c l c(+/-)$ heterozygotes are viable and fertile [31]. Hence, our data also support that GSH synthesis is essential for early mouse development. GSH is particularly critical for normal embryonic development of the visceral yolk sac or the ectoplacental cone, highly vascularized tissues [31]. Of interest, other tissue specific Gclc-null homozygous mice have been characterized, including those targeting the epidermis and liver $[25,36]$. Keratinocyte-specific deficiency in Gclc caused DNA and mitochondrial damage, as well as a strong reduction in keratinocyte viability, partially affecting the epidermal architecture and healing [36]. Similarly, the selective loss of GSH synthesis in hepatocytes leads to steatosis that may be accompanied by mitochondrial damage and 
A

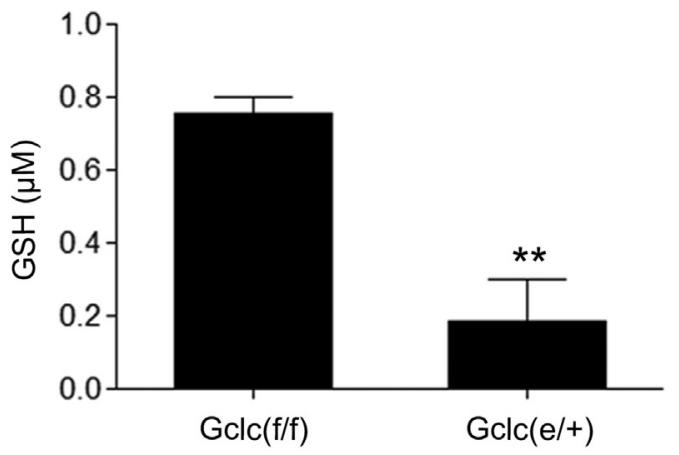

C

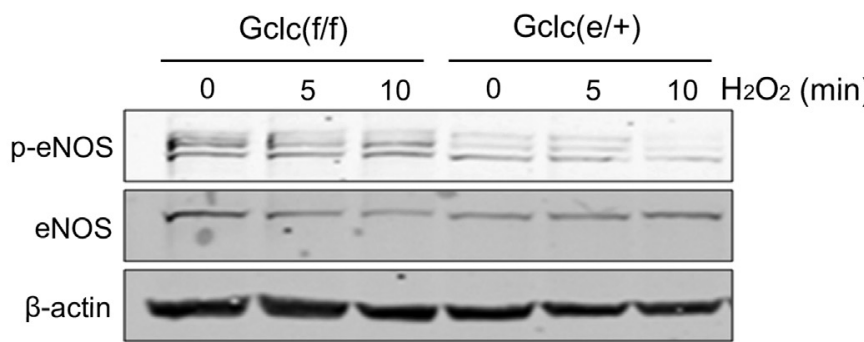

$\mathrm{D}$

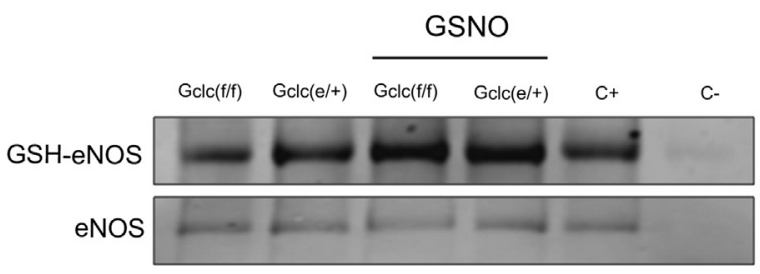

B
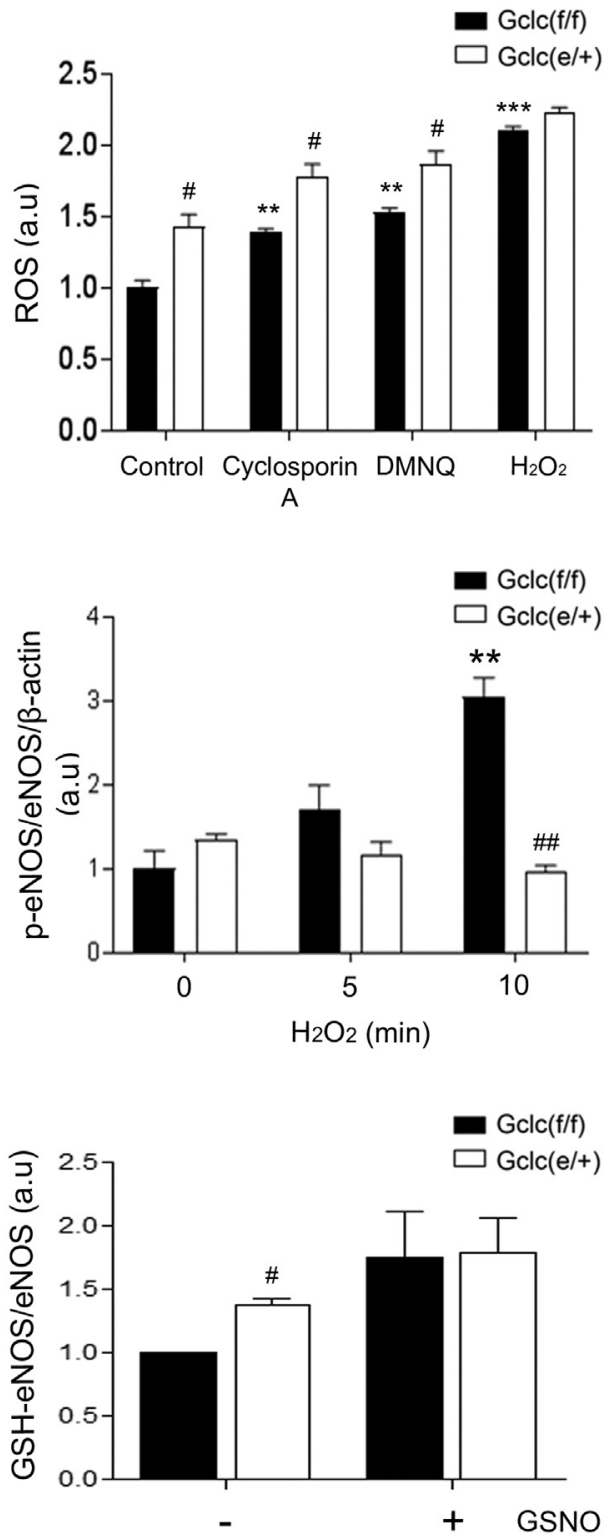

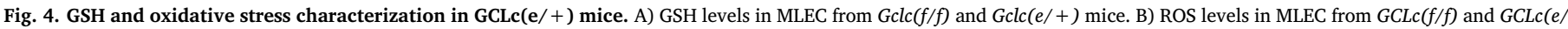

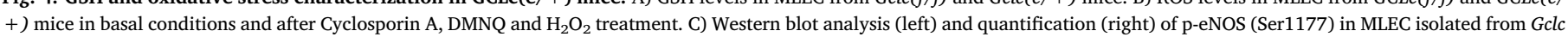

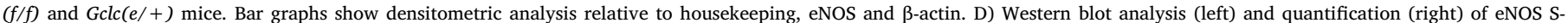

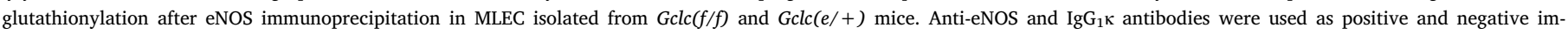

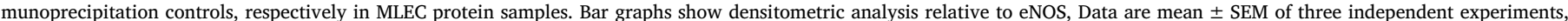



hepatic failure [25].

Our results show that GCLc protein expression was significantly downregulated in approximately $40 \%$ in MLEC derived from haplo-insufficient mice. Of note, this was reflected by $80 \%$ decrease in endothelial GSH levels. Overall, these reductions are higher than those observed in the liver of whole haplo-insufficient mice (20\%) [31], pointing to an enhanced repercussion of the genetic modification within the vascular endothelium. Genetic targeting of the Gclc subunit was not accompanied by compensatory changes in the GCLm subunit. MLEC from $\operatorname{Gclc}(e /+)$ showed increased basal and stimulated ROS levels in response to several electrophilic stimuli, suggesting a weakened endogenous antioxidant response, even in the absence of differences in the level of oxidized GSH. In humans, mutations in the GCLc gene may lead to reduction levels of GSH as low as $2 \%$ of normal in their erythrocytes and affected individuals suffer hemolytic anemia [37]. Furthermore, the $-129 \mathrm{~T}$ polymorphism within the promoter region of the
GCLc gene suppresses GCLC gene induction response to oxidant agents and it correlates with coronary endothelial vasomotor dysfunction [38].

Endothelial nitric oxide synthase (eNOS) is a critical regulator of vascular homeostasis by generating nitric oxide (NO) from L-arginine oxidation. eNOS activity is tightly regulated by posttranslational modifications [39]. Phosphorylation at different sites may have gain or loss functional consequences $[40,41]$. In general, it is accepted that Ser1177 phosphorylation, which may be elicited by the PI3K-Akt signaling pathways, conveys eNOS activation [42]. In opposition, a reduction in the levels of phosphorylation at this Ser-1177 site in response to appropriate stimuli can be interpreted as eNOS inactivation [41]. Of interest, the redox state in the cell is able to regulate eNOS Ser-1177 phosphorylation levels [42]. It has been shown that in endothelial cells under an unbalanced electrophilic tone, eNOS may become uncoupled and transform into a new potential source for the generation of superoxide radical anion $\left(\mathrm{O}_{2}^{-}\right)$. eNOS uncoupling is linked to an increased 

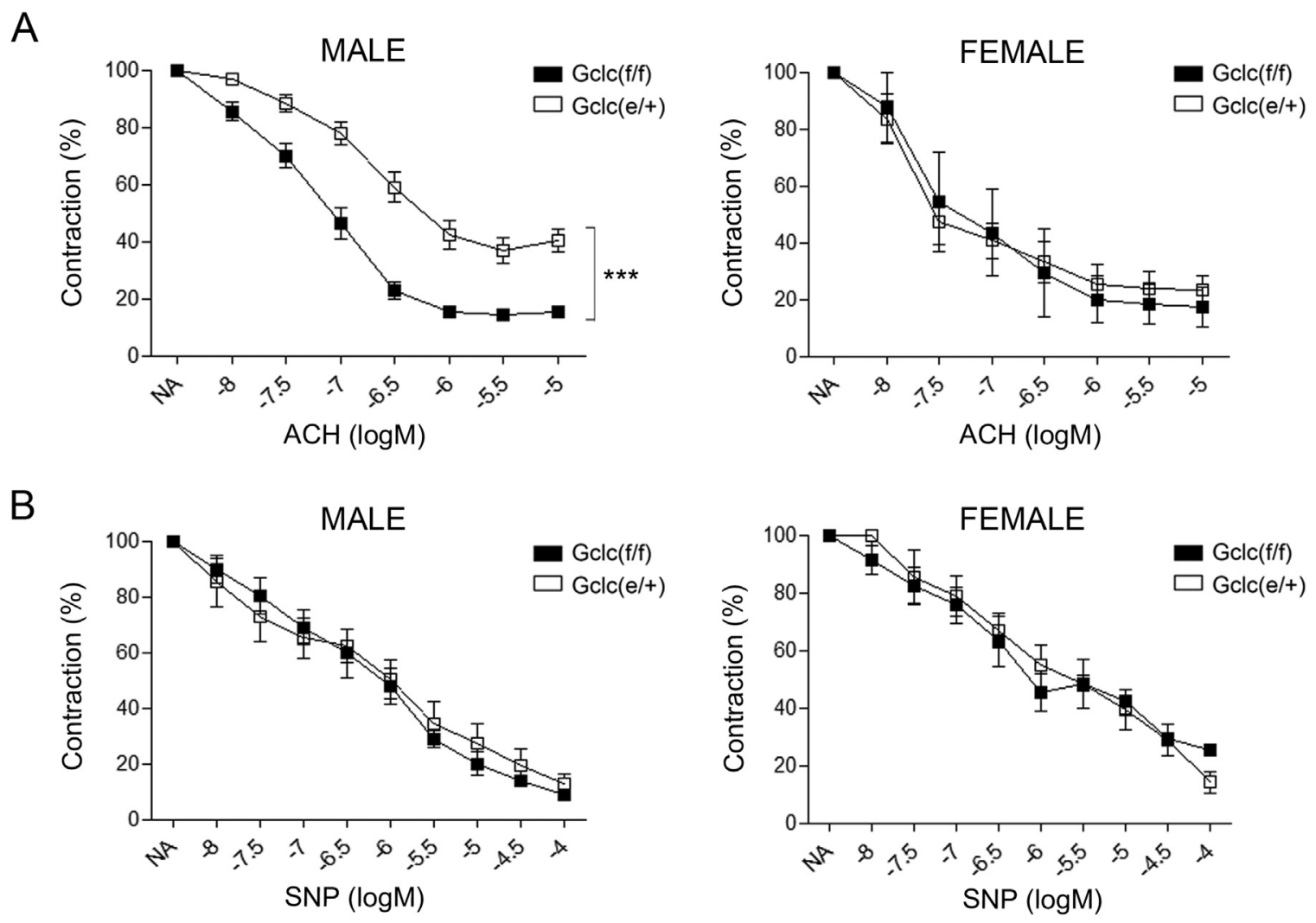

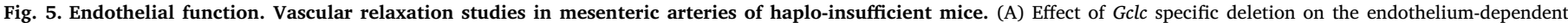

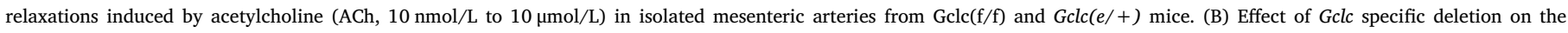

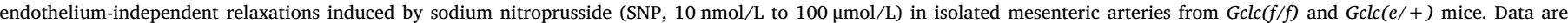

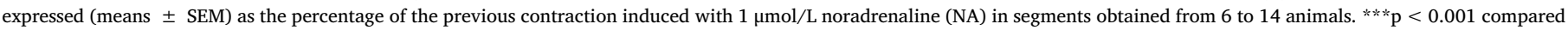
to mesenteric arteries from $\operatorname{Gclc}(f / f)$ mice.

presence of the monomeric form of the enzyme. It is also currently accepted that a critical perturbation leading to its uncoupled state is the relative deficiency of the eNOS catalytic cofactor tetrahydrobiopterin $\left(\mathrm{BH}_{4}\right)$ [10]. Furthermore, eNOS can suffer $S$-glutathionylation at some specific cysteine residues. This mixed disulfide(s) formation has been also proposed as a mechanism for eNOS uncoupling [10]. We have observed that MLEC from endothelial Gclc haplo-insufficient mice showed reduced phosphorylation of eNOS (Ser 1177) and increased eNOS S-glutathionylation, compared to MLEC from wild type (WT) mice. The increased electrophilic tone present in the MLEC from Gclc(e/ +) mice could contribute to both $\mathrm{BH}_{4}$ depletion and consequently increased eNOS $S$-glutathionylation, resulting in an uncoupled state [43]. However, the precise mechanism(s) and order by which these biochemical modifications are occurring in the Gclc haplo-insufficient cells have not been explored in this study and will require further investigation.

The endothelium controls the vascular tone by releasing vasoactive substances in response to physical and chemical signals. eNOS-derived NO has emerged as the most notable endothelium-derived vasodilator. However, perturbations in the vascular tone are germane to vascular pathologies, such as hypertension, vasculitides, atherosclerosis and diabetic vasculopathy [44]. Our studies in mesenteric arteries demonstrated impaired endothelium-dependent vasodilation in $\operatorname{Gclc}(e /+)$ male mice in response to ACh, which was corrected by pre-incubation with $\mathrm{BH}_{4}$ and GSH ethyl-ester. However, no differences were observed in relaxant responses elicited by SNP among $\operatorname{Gclc}(e /+)$ and WT micederived mesenteric vessels, supporting the specificity of the endothelial genetic targeting. Improvement of endothelial vasodilation by pre-incubation with GSH ethyl-ester and $\mathrm{BH}_{4}$ can be attributed to a potentiation of the NO/cGMP pathway-mediated relaxation of smooth muscle since $\mathrm{BH}_{4}$ preserves eNOS dimerization and improves endothelial function through flavin-mediated electron transference [45]. $\mathrm{BH}_{4}$ availability in the mesenteric vascular bed of $G c l c(\mathrm{e} /+)$ male mice can also be hampered by a decreased biosynthetic rate or increased oxidation as previously described [44]. It has been reported that modulation of the Ser1177 phosphorylation site affects endothelium-dependent vasodilation [41]. Furthermore, restoring GSH levels by pre-treatment with GSH-ethyl-ester would reduce oxidative environment in Gclc-deficient mesenteric endothelial and could also enhance the activity of glutathione-dependent enzymes such as glutaredoxins, which have been shown to revert eNOS S-glutathionylation with restoration of NO synthase activity [46]. In our hands the antioxidant molecule NAC, which is expected to increase the nucleophilic tone and can serve as a precursor for GSH, did not significantly revert the impaired endothelium-dependent vasodilation in GCLc $(\mathrm{e} /+)$ male mice, although we observed a tendency to improve impaired relaxation. It is possible that limited permeability of the molecule resulted in only modest enhanced endothelial bioavailability of GSH that was not enough to display a full reversion in the deranged vascular reactivity of Gclc haplo-insufficient mice. Nevertheless, our results support that endothelial dysfunction of $G c l c(e /+)$ mice is associated with reduced GSH bioavailability that can be compensated by improving eNOS function through the addition of critical cofactors.

One striking finding in this study is the observation that there was a gender-related effect in the vascular relaxation response, as Gclc haploinsufficient female mice did not show any difference compared to WT mice. The importance of estrogens on endothelial function has been established since reports of the last century [47]. At the biochemical level early studies showed that estradiol induces eNOS translocation to the cytosol and activation [48]. In addition, estrogens regulate antioxidant enzyme expression and function [48]. Hence, one plausible explanation is that inherent hormonal gender differences could 
A

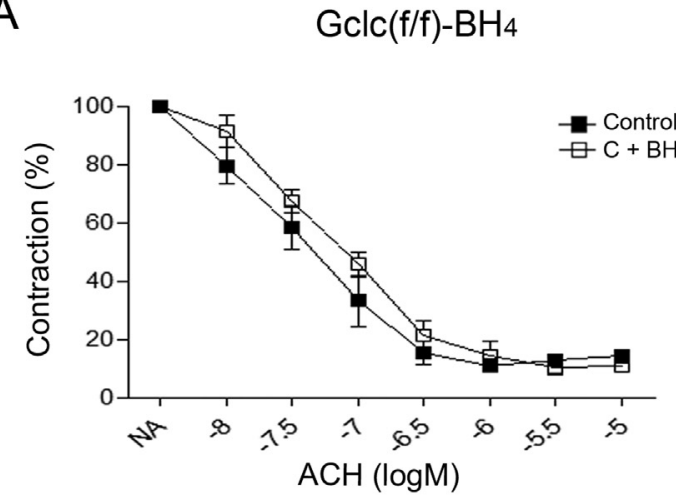

B

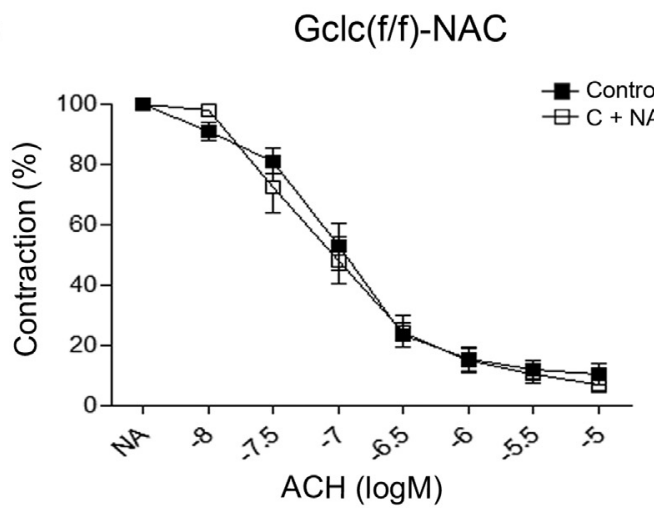

C

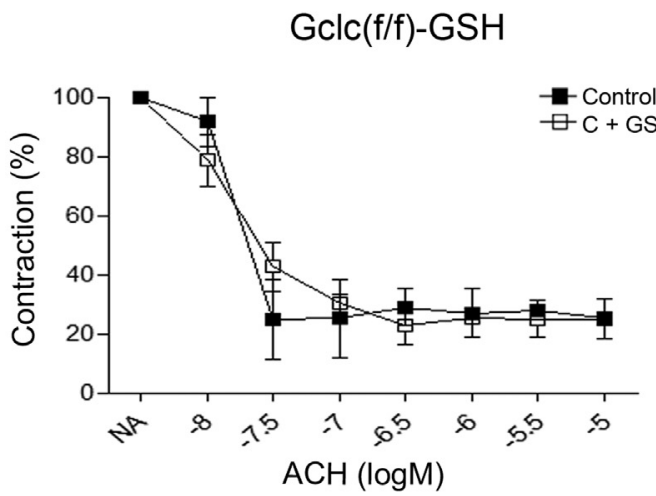

Gclc(e/+)-BH4
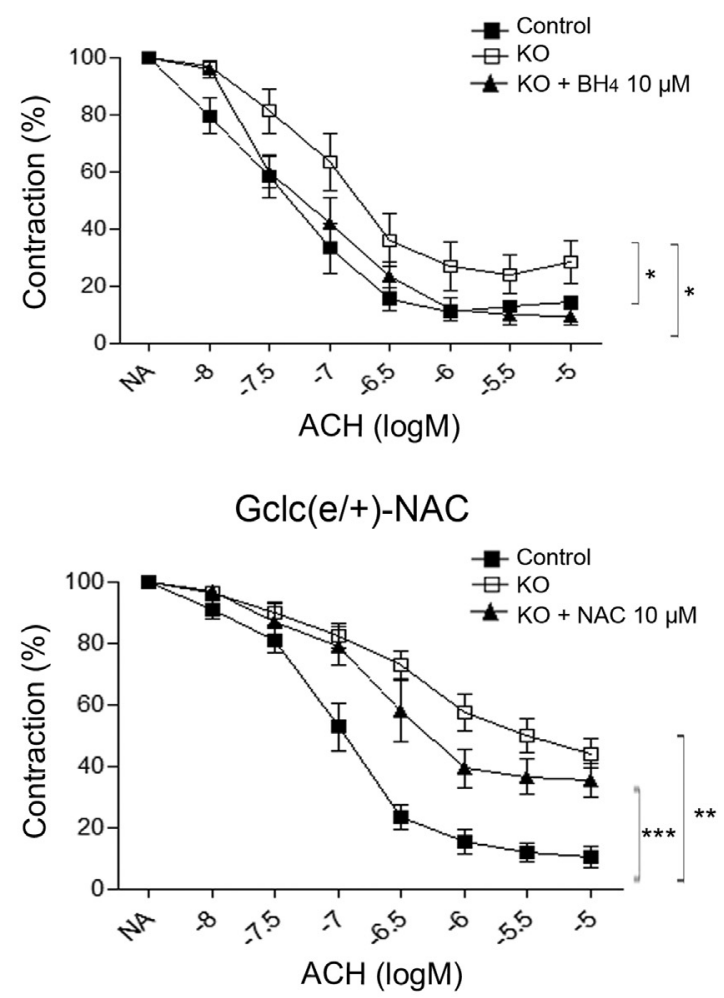

Gclc(e/+)-GSH

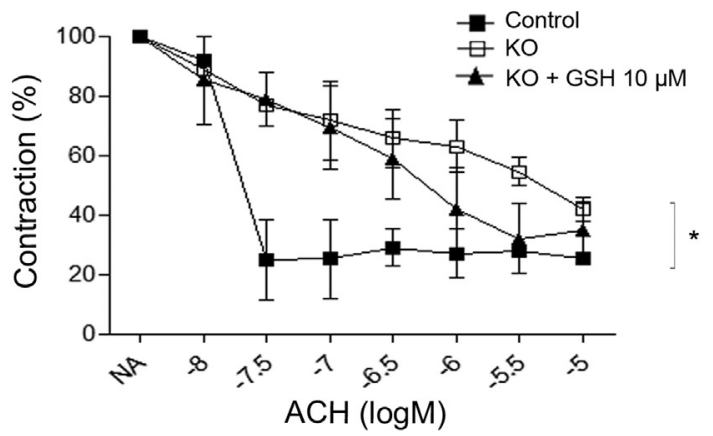

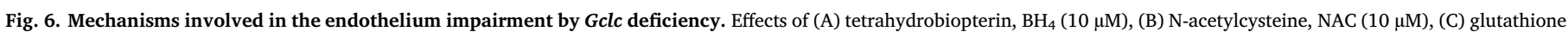

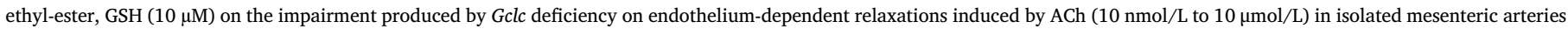

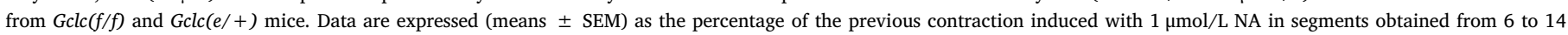

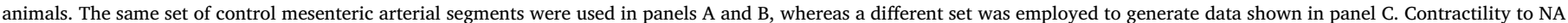
was not statistically different among groups. ${ }^{*} \mathrm{p}<0.05$, ${ }^{* *} \mathrm{p}<0.01$ and ${ }^{* * *} \mathrm{p}<0.001$ compared to mesenteric arteries from $G c l c(f / f)$ mice.

compensate for the reduction of GSH levels in the vascular endothelium of female mice through NO-dependent mechanisms. More recent studies have also pointed to the capacity of estrogens to increase availability of $\mathrm{BH}_{4}$ [49] and also to the capacity of NO and SOD-1 to account for reduced neointimal hyperplasia in female mice [50]. The molecular mechanisms behind the observed differences in our study were not explored. Although, we believe it is sound to hypothesize that they might be related to the profound influence of an estrogenic environment on vascular function through the aforementioned effects.

Whereas in our model we did not observe compensation for the genetic deficiency of Gclc by increased expression of the other component of the enzyme, $\mathrm{Gclm}$, rigorous studies of vascular function performed in the whole null model of Gclm(-/-) shed important light on the consequences of GSH deficiency for endothelial function [18]. These mice had an $80 \%$ and $25 \%$ reduction in aortic GSH levels in the homozygous and heterozygous null mice, respectively. This was accompanied by increased levels of GSSG and altered endothelial-dependent vascular relaxation. Strikingly, relaxation for higher concentrations of acetylcholine was improved in homozygous $\mathrm{Gclm}$ null mice compared to control or heterozygous animals. Our study underscores that the approach of selectively targeting the endothelium incorporates added value to understand the role of endothelial GSH homeostasis and prevents any confounding factor inherent to total tissular suppression of a key GSH biosynthetic pathway.

Kidney fibrosis is the final outcome of many prevalent renal diseases, such as hypertensive nephropathy, diabetes mellitus and aging. The establishment of fibrosis leads eventually to chronic kidney disease or end-stage renal disease [51]. Transforming growth factor beta (TGF$\beta$ ) is the one of the most potent and ubiquitous fibrogenic cytokines and its increased expression is present in almost all types of organ fibrosis and in different models of experimental fibrosis including UUO. It is accepted that there is a bidirectional relationship between the redox 
A
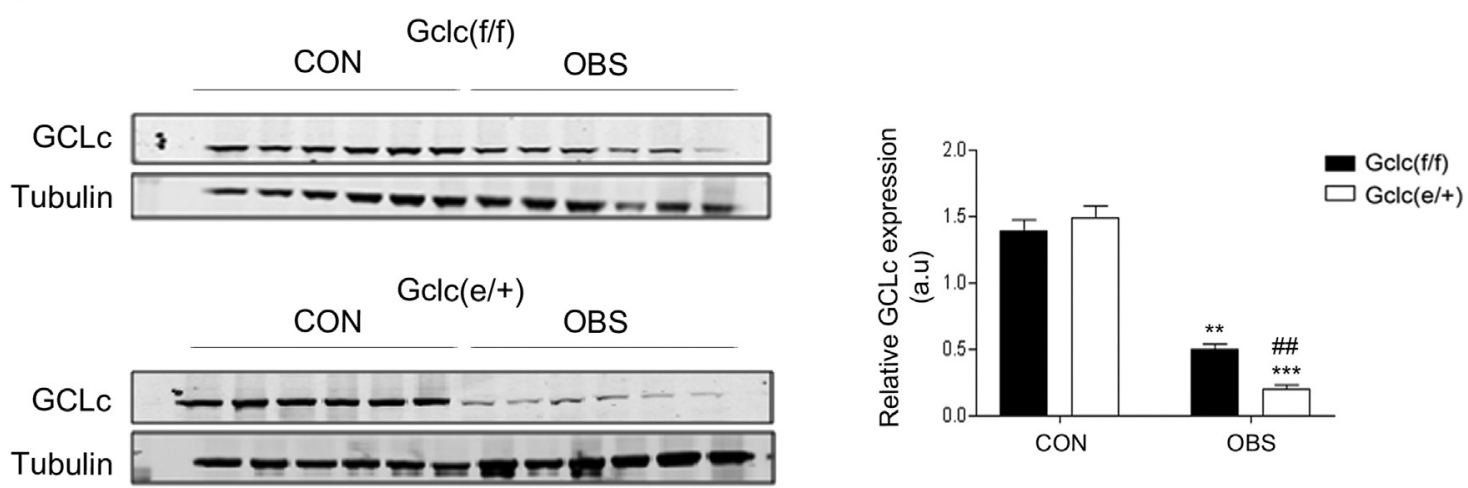

B

C
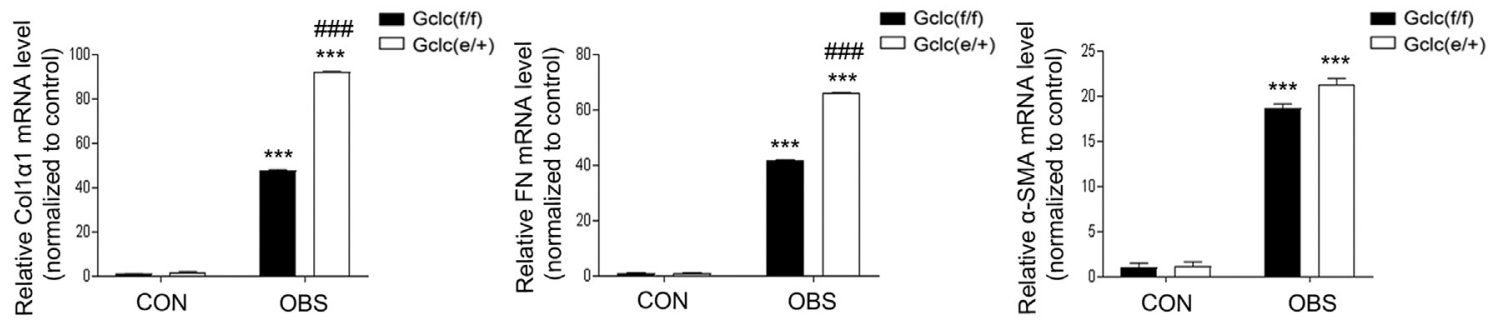

$E$
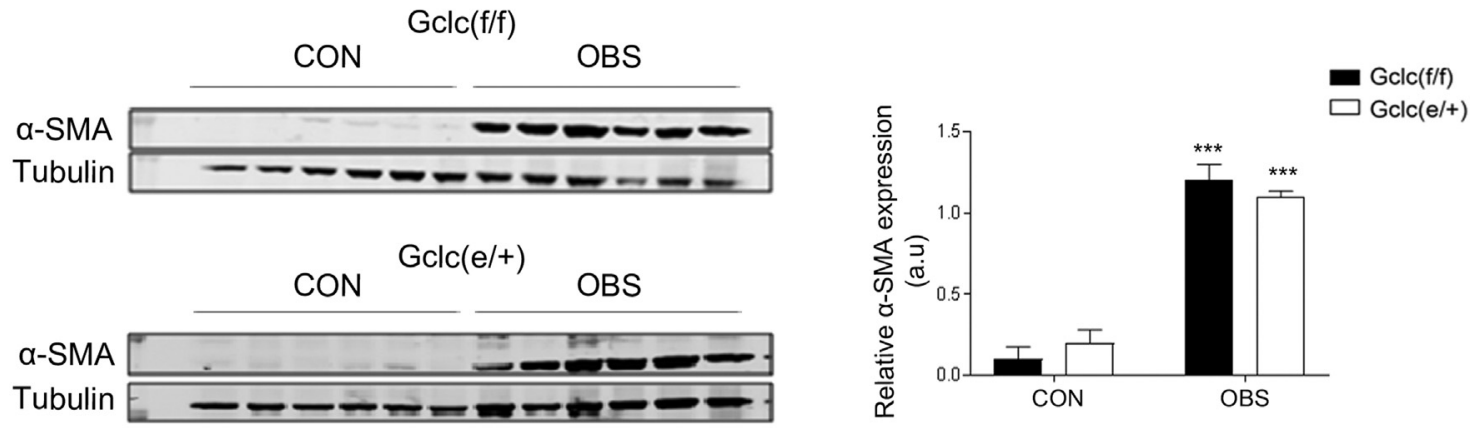

$\mathrm{F}$

G
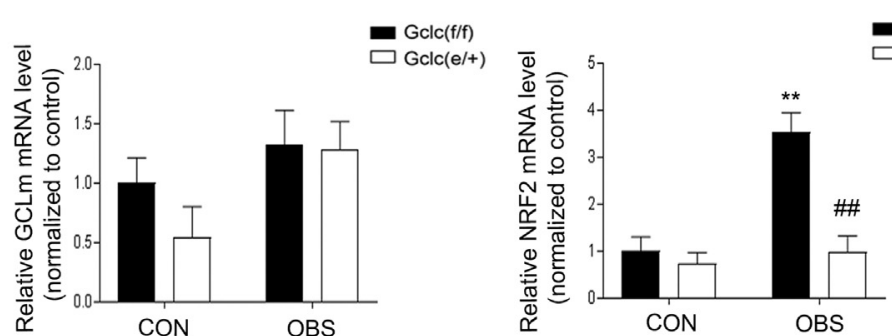

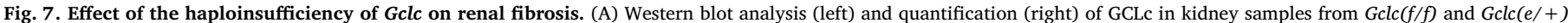

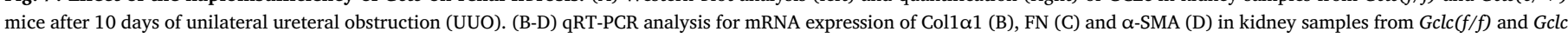

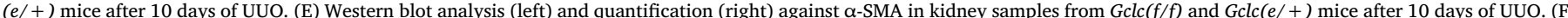

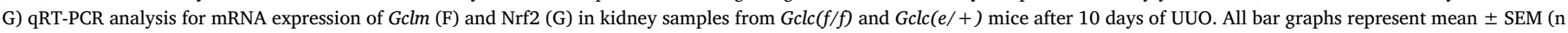

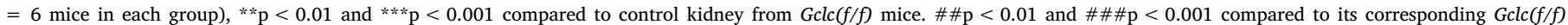
condition.

state and TGF- $\beta$ activation [24]. The homeostatic role provided by the redox pair GSH/GSSG is essential for the redox-related pathophysiological mechanisms underlying fibrosis. The concentration of GSH decreases in experimental fibrosis models, in human fibrotic diseases and several cell types including endothelial cells, hepatocytes, epithelial cells, and fibroblasts, whereas GSH replenishment suppresses TGF- 
A

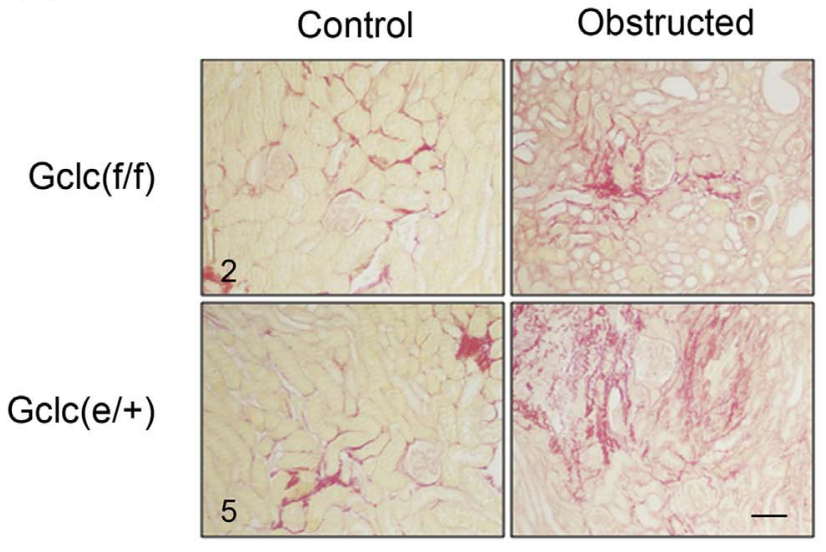

B

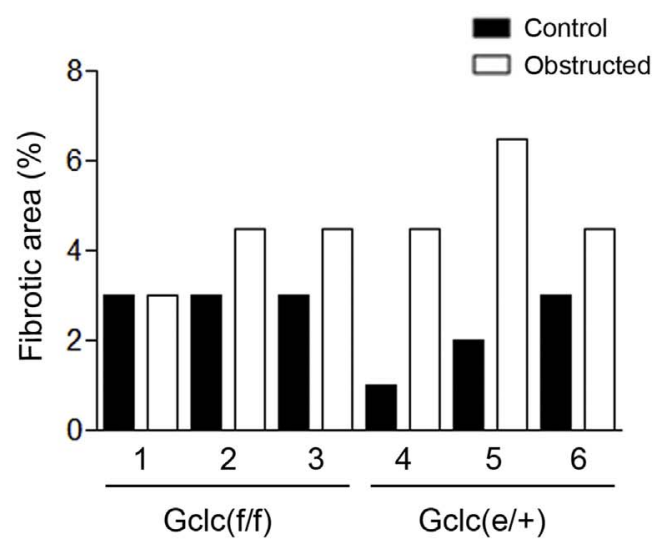

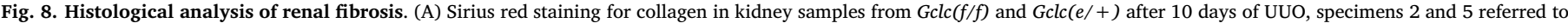
(B). The black bar scale represents $0.1 \mathrm{~mm}$. (B) Quantitative evaluation of kidney fibrosis from $3 \mathrm{Gclc}(f / f)(1-3)$ and $3 \mathrm{Gclc}(e /+)(4-6)$ mice, calculated as the \% of fibrotic area.

$\beta$ 's fibrogenic activity [14]. Endothelial dysfunction is a multifaceted condition that plays a central role in complications related to kidney diseases through the regulation of oxygen levels, the inflammatory response, cellular crosstalk and the generation of renal mesenchymal cells. To study the contribution of endothelial GSH synthesis to renal fibrosis we employed the UUO model in WT and $\operatorname{Gclc}(e /+)$ mice. We observed that obstructed kidneys from these mice exhibited increased deposition of fibrotic markers and an enhanced pattern of interstitial fibrosis as demonstrated by Sirius Red staining (Fig. 8A), in comparison to WT mice. Even though the fibrotic phenotype was only moderately potentiated by the endothelial haplo-insufficiency, it is important to keep in mind that GSH synthesis was only targeted in endothelial cells, while the potential source of myofibroblasts (pericytes, resident fibroblasts, epithelial cells) are predicted to maintain intact redox homeostasis. It is precisely this circumstance that confers significance to our observations, as the vascular endothelium represents only a minor portion of the whole kidney tissue albeit the specific deficit of GSH appears to be important for the fibrotic response. However, the fact that we used the Tie2-Cre model to generate the GCLc null mice poses a limitation to our study as, in addition to its expression in endothelial cells, Tie2 has been also described in certain hematopoietic cell lines $[52,53]$. The first stages of fibrogenesis are characterized by a marked inflammatory response. In this regard, an unbalance in macrophage type populations and an active role of monocytes in angiotensin IImediated vascular dysfunction and fibrogenesis have been reported $[54,55]$. The homeostasis of GSH is critical for immune cell $\mathrm{f}$ unction and several reports have illustrated this fact, in particular in the context of atherogenesis and T-cell activation [56-58] In our endothelial dysfunction model, due to the nature of the endothelial reactivity experiments it is reasonable to assume a limited contribution of the immune response to the endothelial relaxation impairment. Nevertheless, in the case of renal injury associated to the UUO model, macrophage activation of the proinflammatory M1 population and recruitment of immune (mainly $\mathrm{T}$ ) cells play an active role. In these conditions, the inflammatory cytokines in the fibrotic milieu lead to an increased production of profibrotic mediators such as TGF- $\beta$ [59]. As stated above, an unbalance in the oxidative stress levels in macrophages can promote the polarization towards an M1 proinflammatory and profibrotic phenotype. Therefore GSH depletion in immune cells would also enhance renal fibrosis progression. Since infiltrating monocytes/macrophages negatively affect vascular function, we may expect an ulterior crosstalk between the immune and endothelial population that could contribute to perpetuate fibrogenesis. All these are important avenues to be explored in future work.

The maintenance of adequate nucleophilic power provided by GSH is essential to prevent fibrogenesis and the molecular bases for this are well established. Jardine et al. reported that TGF- $\beta$-mediates GCLc regulation. TGF- $\beta$ suppressed GCLc promoter activity through the increased binding of c-Jun and Fra-1 dimers to the AP-1 cis-regulatory site within the antioxidant response element (ARE) [22]. In keeping, NAC and GSH showed a clear inhibitory effect on the development of fibrosis in both patients and animal models [60]. In kidney injury, the redox regulation of fibrogenic pathways is related to several mechanisms including the state of oxidation of critical protein thiols [61]. Thus, the disturbance of redox homeostasis in the kidney endothelial compartment is likely to contribute to fibrogenesis as an intact endothelial function is considered indispensable for mounting an appropriate tissue repair response $[62,63]$. In the context of the antioxidant response the Keap1-Nrf2 system has been proposed to be a therapeutic target for renal protection [64]. Nrf2 is a key regulator of antioxidant defense system that modulates the expression of several genes in response to electrophilic stress including phase 2 response genes and antioxidant enzymes such as GCL $[23,65]$. The activation of Nrf2 after the UUO has been invoked as an attenuating mechanism for the development of renal fibrosis [66]. Our data confirmed our previous reported observation whereby UUO-induced renal fibrosis is associated with Gclc downregulation [23], which was even more pronounced in kidneys from haplo-insufficient mice (Fig. 7A). Whereas UUO was able to trigger an up-regulation of Nrf2 levels in WT mice, fibrotic kidneys from $\operatorname{Gclc}(e /+)$ mice failed to do so (Fig. $7 \mathrm{G}$ ). A clear explanation for this observation is not obvious as a reduction of GSH levels should be expected to induce a compensatory up-regulation of the antioxidant response by increasing Nrf2 levels. However, the interplay of TGF- $\beta$ regulation with GSH is a complex one [67]. Moreover, the fact that only endothelial cells were targeted for GCLc, precludes a clear-cut interpretation of these results.

In conclusion, our data establish that the specific endothelial reduction of GSH levels increased basal and stimulated ROS levels, reduced phosphorylation of eNOS (Ser1177) and increased eNOS S-glutathionylation, subsequently impairing endothelium-dependent vasodilation and promoting renal fibrosis following UUO. Thus, we propose that endothelial GSH plays a critical role in endothelial function and the anti-fibrotic response in the kidney.

\section{Acknowledgements}

This work was supported by grants from the Ministerio de Economía y Competitividad (MINECO) SAF2012-31338, SAF2014-52762-R, SAF2015-66107-R also supported by European Regional Development Funds (ERDF-FEDER), and CSD 2007-00020, Instituto de Salud Carlos III REDinREN RD12/0021/0009, RD16/0009/0016, Comunidad de Madrid "Fibroteam" S2010/BMD-2321, and Fundación Renal "Iñigo 
Alvarez de Toledo," all from Spain. This study was supported by the European Cooperation in Science and Research COST actions BM-1203 (EU-ROS) and BM-1005 (ENOGAS). The CBMSO receives institutional support from Fundación "Ramón Areces". Verónica Miguel is currently supported by the MINECO program of Formación de Personal Investigador (FPI BES-2013-065986). Cristina Espinosa-Diez was a fellow of the FPI program from MINECO (BES-2010-035389).

\section{References}

[1] A. Daiber, S. Steven, A. Weber, V.V. Shuvaev, V.R. Muzykantov, I. Laher, H. Li, S. Lamas, T. Munzel, Targeting vascular (endothelial) dysfunction, Br. J. Pharmacol. 174 (2017) 1591-1619.

[2] E. Schulz, E. Anter, J.F. Keaney Jr., Oxidative stress, antioxidants, and endothelial function, Curr. Med. Chem. 11 (2004) 1093-1104.

[3] U. Forstermann, Oxidative stress in vascular disease: causes, defense mechanisms and potential therapies, Nat. Clin. Pract. Cardiovasc. Med. 5 (2008) 338-349.

[4] C. Espinosa-Diez, V. Miguel, D. Mennerich, T. Kietzmann, P. Sanchez-Perez, S. Cadenas, S. Lamas, Antioxidant responses and cellular adjustments to oxidative stress, Redox Biol. 6 (2015) 183-197.

[5] H. Steinbrenner, H. Sies, Protection against reactive oxygen species by selenoproteins, Biochim. Biophys. Acta 1790 (2009) 1478-1485.

[6] D.A. Dickinson, D.R. Moellering, K.E. Iles, R.P. Patel, A.L. Levonen, A. Wigley, V.M. Darley-Usmar, H.J. Forman, Cytoprotection against oxidative stress and the regulation of glutathione synthesis, Biol. Chem. 384 (2003) 527-537.

[7] O.W. Griffith, Biologic and pharmacologic regulation of mammalian glutathione synthesis, Free Radic. Biol. Med. 27 (1999) 922-935.

[8] C.C. Franklin, D.S. Backos, I. Mohar, C.C. White, H.J. Forman, T.J. Kavanagh, Structure, function, and post-translational regulation of the catalytic and modifier subunits of glutamate cysteine ligase, Mol. Asp. Med. 30 (2009) 86-98.

[9] Y. Chen, H.G. Shertzer, S.N. Schneider, D.W. Nebert, T.P. Dalton, Glutamate cysteine ligase catalysis: dependence on ATP and modifier subunit for regulation of tissue glutathione levels, J. Biol. Chem. 280 (2005) 33766-33774.

[10] C.A. Chen, T.Y. Wang, S. Varadharaj, L.A. Reyes, C. Hemann, M.A. Talukder, Y.R. Chen, L.J. Druhan, J.L. Zweier, S-glutathionylation uncouples eNOS and reg ulates its cellular and vascular function, Nature 468 (2010) 1115-1118.

[11] L. Yuan, N. Kaplowitz, Glutathione in liver diseases and hepatotoxicity, Mol. Asp. Med. 30 (2009) 29-41.

[12] I. Hakki Kalkan, M. Suher, The relationship between the level of glutathione, impairment of glucose metabolism and complications of diabetes mellitus, Pak. J. Med. Sci. 29 (2013) 938-942.

[13] R. Dringen, Metabolism and functions of glutathione in brain, Prog. Neurobiol. 62 (2000) 649-671.

[14] R.M. Liu, K.A. Gaston Pravia, Oxidative stress and glutathione in TGF-beta-mediated fibrogenesis, Free Radic. Biol. Med. 48 (2010) 1-15.

[15] B.J. Mills, M.M. Weiss, C.A. Lang, M.C. Liu, C. Ziegler, Blood glutathione and cysteine changes in cardiovascular disease, J. Lab. Clin. Med. 135 (2000) 396-401.

[16] L.M. Bekris, C. Shephard, M. Peterson, J. Hoehna, B. Van Yserloo, E. Rutledge, F. Farin, T.J. Kavanagh, A. Lernmark, Glutathione-s-transferase M1 and T1 polymorphisms and associations with type 1 diabetes age-at-onset, Autoimmunity 38 (2005) 567-575.

[17] S.N. Nichenametla, P. Lazarus, J.P. Richie Jr., A GAG trinucleotide-repeat poly morphism in the gene for glutathione biosynthetic enzyme, GCLC, affects gene expression through translation, FASEB J. 25 (2011) 2180-2187.

[18] C.S. Weldy, I.P. Luttrell, C.C. White, V. Morgan-Stevenson, T.K. Bammler, R.P. Beyer, Z. Afsharinejad, F. Kim, K. Chitaley, T.J. Kavanagh, Glutathione (GSH) and the GSH synthesis gene Gclm modulate vascular reactivity in mice, Free Radic. Biol. Med. 53 (2012) 1264-1278.

[19] J. Lim, B.N. Nakamura, I. Mohar, T.J. Kavanagh, U. Luderer, Glutamate cysteine ligase modifier subunit ( $\mathrm{Gclm}$ ) null mice have increased ovarian oxidative stress and accelerated age-related ovarian failure, Endocrinology 156 (2015) 3329-3343.

[20] L.A. McConnachie, D. Botta, C.C. White, C.S. Weldy, H.W. Wilkerson, J. Yu, R. Dills, X. Yu, W.C. Griffith, E.M. Faustman, F.M. Farin, S.E. Gill, W.C. Parks, X. Hu, X. Gao, D.L. Eaton, T.J. Kavanagh, The glutathione synthesis gene Gclm modulates amphiphilic polymer-coated CdSe/ZnS quantum dot-induced lung inflammation in mice, PLoS One 8 (2013) e64165.

[21] L.P. Liang, T.J. Kavanagh, M. Patel, Glutathione deficiency in Gclm null mice results in complex I inhibition and dopamine depletion following paraquat administration, Toxicol. Sci. 134 (2013) 366-373.

[22] H. Jardine, W. MacNee, K. Donaldson, I. Rahman, Molecular mechanism of trans forming growth factor (TGF)-beta1-induced glutathione depletion in alveolar epithelial cells. Involvement of AP-1/ARE and Fra-1, J. Biol. Chem. 277 (2002) 21158-21166.

[23] C. Espinosa-Diez, M. Fierro-Fernandez, F. Sanchez-Gomez, F. Rodriguez-Pascual, M. Alique, M. Ruiz-Ortega, N. Beraza, M.L. Martinez-Chantar, C. FernandezHernando, S. Lamas, Targeting of gamma-glutamyl-cysteine ligase by miR-433 reduces glutathione biosynthesis and promotes TGF-beta-dependent fibrogenesis, Antioxid. Redox Signal. 23 (2015) 1092-1105.

[24] R.M. Liu, L.P. Desai, Reciprocal regulation of TGF-beta and reactive oxygen species: a perverse cycle for fibrosis, Redox Biol. 6 (2015) 565-577.

[25] Y. Chen, Y. Yang, M.L. Miller, D. Shen, H.G. Shertzer, K.F. Stringer, B. Wang, S.N. Schneider, D.W. Nebert, T.P. Dalton, Hepatocyte-specific Gclc deletion leads to rapid onset of steatosis with mitochondrial injury and liver failure, Hepatology 45
(2007) 1118-1128.

[26] K.J. Livak, T.D. Schmittgen, Analysis of relative gene expression data using real time quantitative PCR and the 2(-Delta Delta C(T)) method, Methods 25 (2001) 402-408.

[27] L. Rodriguez-Manas, J. Angulo, C. Peiro, J.L. Llergo, A. Sanchez-Ferrer, P. LopezDoriga, C.F. Sanchez-Ferrer, Endothelial dysfunction and metabolic control in streptozotocin-induced diabetic rats, Br. J. Pharmacol. 123 (1998) 1495-1502.

[28] R.L. Chevalier, M.S. Forbes, B.A. Thornhill, Ureteral obstruction as a model of renal interstitial fibrosis and obstructive nephropathy, Kidney Int. 75 (2009) 1145-1152.

[29] J.M. Street, A.C. Souza, A. Alvarez-Prats, T. Horino, X. Hu, P.S. Yuen, R.A. Star, Automated quantification of renal fibrosis with Sirius Red and polarization contrast microscopy, Physiol. Rep. 2 (2014).

[30] A. Forde, R. Constien, H.J. Grone, G. Hammerling, B. Arnold, Temporal Cremediated recombination exclusively in endothelial cells using Tie2 regulatory elements, Genesis 33 (2002) 191-197.

[31] T.P. Dalton, M.Z. Dieter, Y. Yang, H.G. Shertzer, D.W. Nebert, Knockout of the mouse glutamate cysteine ligase catalytic subunit (Gclc) gene: embryonic lethal when homozygous, and proposed model for moderate glutathione deficiency when heterozygous, Biochem. Biophys. Res. Commun. 279 (2000) 324-329.

[32] M. Redondo-Horcajo, N. Romero, P. Martinez-Acedo, A. Martinez-Ruiz, C. Quijano, C.F. Lourenco, N. Movilla, J.A. Enriquez, F. Rodriguez-Pascual, E. Rial, R. Radi, J. Vazquez, S. Lamas, Cyclosporine A-induced nitration of tyrosine 34 MnSOD in endothelial cells: role of mitochondrial superoxide, Cardiovasc. Res. 87 (2010) $356-365$.

[33] J.L. Zweier, C.A. Chen, L.J. Druhan, S-glutathionylation reshapes our understanding of endothelial nitric oxide synthase uncoupling and nitric oxide/reactive oxygen species-mediated signaling, Antioxid. Redox Signal. 14 (2011) 1769-1775.

[34] D. Fulton, J. Fontana, G. Sowa, J.P. Gratton, M. Lin, K.X. Li, B. Michell, B.E. Kemp, D. Rodman, W.C. Sessa, Localization of endothelial nitric-oxide synthase phosphorylated on serine 1179 and nitric oxide in Golgi and plasma membrane defines the existence of two pools of active enzyme, J. Biol. Chem. 277 (2002) 4277-4284.

[35] M.J. Crabtree, R. Brixey, H. Batchelor, A.B. Hale, K.M. Channon, Integrated redox sensor and effector functions for tetrahydrobiopterin- and glutathionylation-dependent endothelial nitric-oxide synthase uncoupling, J. Biol. Chem. 288 (2013) $561-569$.

[36] M. Telorack, M. Meyer, I. Ingold, M. Conrad, W. Bloch, S. Werner, A glutathioneNrf2-thioredoxin cross-talk ensures keratinocyte survival and efficient wound repair, PLoS Genet. 12 (2016) e1005800.

[37] E. Ristoff, C. Augustson, J. Geissler, T. de Rijk, K. Carlsson, J.L. Luo, K. Andersson, R.S. Weening, R. van Zwieten, A. Larsson, D. Roos, A missense mutation in the heavy subunit of gamma-glutamylcysteine synthetase gene causes hemolytic anemia, Blood 95 (2000) 2193-2196.

[38] S. Koide, K. Kugiyama, S. Sugiyama, S. Nakamura, H. Fukushima, O. Honda, M. Yoshimura, H. Ogawa, Association of polymorphism in glutamate-cysteine ligase catalytic subunit gene with coronary vasomotor dysfunction and myocardial infarction, J. Am. Coll. Cardiol. 41 (2003) 539-545.

[39] L.J. Ignarro, Nitric oxide as a unique signaling molecule in the vascular system: a historical overview, J. Physiol. Pharmacol. 53 (2002) 503-514.

[40] I. Fleming, Molecular mechanisms underlying the activation of eNOS, Pflug. Arch. 459 (2010) 793-806.

[41] G.K. Kolluru, J.H. Siamwala, S. Chatterjee, eNOS phosphorylation in health and disease, Biochimie 92 (2010) 1186-1198.

[42] D. Fulton, J.P. Gratton, T.J. McCabe, J. Fontana, Y. Fujio, K. Walsh, T.F. Franke, A. Papapetropoulos, W.C. Sessa, Regulation of endothelium-derived nitric oxide production by the protein kinase Akt, Nature 399 (1999) 597-601.

[43] D.M. Townsend, S-glutathionylation: indicator of cell stress and regulator of the unfolded protein response, Mol. Interv. 7 (2007) 313-324.

[44] B. Lassegue, K.K. Griendling, NADPH oxidases: functions and pathologies in the vasculature, Arterioscler. Thromb. Vasc. Biol. 30 (2010) 653-661.

[45] S. Chuaiphichai, M.J. Crabtree, E. McNeill, A.B. Hale, L. Trelfa, K.M. Channon, G. Douglas, A key role for tetrahydrobiopterin-dependent endothelial NOS regulation in resistance arteries: studies in endothelial cell tetrahydrobiopterin-deficient mice, Br. J. Pharmacol. 174 (2017) 657-671.

[46] T. Sugiyama, T. Michel, Thiol-metabolizing proteins and endothelial redox state: differential modulation of eNOS and biopterin pathways, Am. J. Physiol. Heart Circ. Physiol. 298 (2010) H194-H201.

[47] C.P. Weiner, I. Lizasoain, S.A. Baylis, R.G. Knowles, I.G. Charles, S. Moncada, Induction of calcium-dependent nitric oxide synthases by sex hormones, Proc. Natl. Acad. Sci. USA 91 (1994) 5212-5216.

[48] R.M. Goetz, H.S. Thatte, P. Prabhakar, M.R. Cho, T. Michel, D.E. Golan, Estradiol induces the calcium-dependent translocation of endothelial nitric oxide synthase, Proc. Natl. Acad. Sci. USA 96 (1999) 2788-2793.

[49] K.K. Lam, Y.M. Lee, G. Hsiao, S.Y. Chen, M.H. Yen, Estrogen therapy replenishes vascular tetrahydrobiopterin and reduces oxidative stress in ovariectomized rats, Menopause 13 (2006) 294-302.

[50] R.C. Morales, E.S. Bahnson, G.E. Havelka, N. Cantu-Medellin, E.E. Kelley, M.R. Kibbe, Sex-based differential regulation of oxidative stress in the vasculature by nitric oxide, Redox Biol. 4 (2015) 226-233.

[51] A.B. Fogo, Mechanisms of progression of chronic kidney disease, Pediatr. Nephrol. 22 (2007) 2011-2022.

[52] E. Kukk, U. Wartiovaara, Y. Gunji, J. Kaukonen, H.J. Buhring, I. Rappold, M.T. Matikainen, P. Vihko, J. Partanen, A. Palotie, K. Alitalo, R. Alitalo, Analysis of Tie receptor tyrosine kinase in haemopoietic progenitor and leukaemia cells, Br. J. Haematol. 98 (1997) 195-203.

[53] N. Jones, K. Iljin, D.J. Dumont, K. Alitalo, Tie receptors: new modulators of angiogenic and lymphangiogenic responses, Nat. Rev. Mol. Cell Biol. 2 (2001) 
$257-267$.

[54] X. Wang, M. Hausding, S.Y. Weng, Y.O. Kim, S. Steven, T. Klein, A. Daiber, D. Schuppan, Gliptins suppress inflammatory macrophage activation to mitigate inflammation, fibrosis, oxidative stress, and vascular dysfunction in models of nonalcoholic steatohepatitis and liver fibrosis, Antioxid. Redox Signal. (2017).

[55] P. Wenzel, M. Knorr, S. Kossmann, J. Stratmann, M. Hausding, S. Schuhmacher, S.H. Karbach, M. Schwenk, N. Yogev, E. Schulz, M. Oelze, S. Grabbe, H. Jonuleit, C. Becker, A. Daiber, A. Waisman, T. Munzel, Lysozyme M-positive monocytes mediate angiotensin II-induced arterial hypertension and vascular dysfunction, Circulation 124 (2011) 1370-1381.

[56] H.S. Kim, S.L. Ullevig, D. Zamora, C.F. Lee, R. Asmis, Redox regulation of MAPK phosphatase 1 controls monocyte migration and macrophage recruitment, Proc. Natl. Acad. Sci. USA 109 (2012) E2803-E2812.

[57] M. Qiao, M. Kisgati, J.M. Cholewa, W. Zhu, E.J. Smart, M.S. Sulistio, R. Asmis, Increased expression of glutathione reductase in macrophages decreases atherosclerotic lesion formation in low-density lipoprotein receptor-deficient mice, Arterioscler. Thromb. Vasc. Biol. 27 (2007) 1375-1382.

[58] L.K. Sha, W. Sha, L. Kuchler, A. Daiber, A.K. Giegerich, A. Weigert, T. Knape, R. Snodgrass, K. Schroder, R.P. Brandes, B. Brune, A. von Knethen, Loss of Nrf2 in bone marrow-derived macrophages impairs antigen-driven CD8(+) T cell function by limiting GSH and Cys availability, Free Radic. Biol. Med 83 (2015) 77-88.

[59] T. Vanhove, R. Goldschmeding, D. Kuypers, Kidney fibrosis: origins and interventions, Transplantation 101 (2017) 713-726.
[60] M. Myllarniemi, R. Kaarteenaho, Pharmacological treatment of idiopathic pulmonary fibrosis - preclinical and clinical studies of pirfenidone, nintedanib, and Nacetylcysteine, Eur. Clin. Respir. J. 2 (2015).

[61] D.M. Okamura, S. Pennathur, The balance of powers: redox regulation of fibrogenic pathways in kidney injury, Redox Biol. 6 (2015) 495-504.

[62] M.A. Gonzalez, A.P. Selwyn, Endothelial function, inflammation, and prognosis in cardiovascular disease, Am. J. Med 115 (Suppl. 8A) (2003) 99S-106S.

[63] M.D. Ross, E. Malone, G. Florida-James, Vascular ageing and exercise: focus on cellular reparative processes, Oxid. Med. Cell Longev. 2016 (2016) 3583956.

[64] M. Nezu, N. Suzuki, M. Yamamoto, Targeting the KEAP1-NRF2 system to prevent kidney disease progression, Am. J. Nephrol. 45 (2017) 473-483.

[65] A.A. Shokeir, N. Barakat, A.M. Hussein, A. Awadalla, A.M. Harraz, S. Khater, K. Hemmaid, A.I. Kamal, Activation of Nrf2 by ischemic preconditioning and sulforaphane in renal ischemia/reperfusion injury: a comparative experimental study, Physiol. Res. 64 (2015) 313-323.

[66] S. Chung, S. Kim, M. Kim, E.S. Koh, H.E. Yoon, H.S. Kim, C.W. Park, Y.S. Chang, S.J. Shin, T-type calcium channel blocker attenuates unilateral ureteral obstructioninduced renal interstitial fibrosis by activating the Nrf2 antioxidant pathway, Am. J Transl. Res. 8 (2016) 4574-4585.

[67] I.G. Ryoo, H. Ha, M.K. Kwak, Inhibitory role of the KEAP1-NRF2 pathway in TGFbeta1-stimulated renal epithelial transition to fibroblastic cells: a modulatory effect on SMAD signaling, PLoS One 9 (2014) e93265. 